\title{
Comment on "Effect of competence health cultivation on the prevention and control of inadvertent perioperative hypothermia"

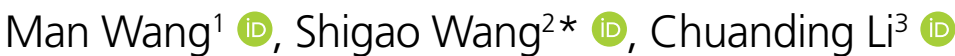

Dear Editor,

We are very pleased to read the study by Wang $R$ and his colleagues ${ }^{1}$ in which they demonstrated that the medical staff can improve the quality of inadvertent perioperative hypothermia (IPH) prevention and management by health competence cultivation and feasible health management measures. However, there are still some issues that should be addressed according to my opinion.

To begin with, in the prospective nursing observation method section, this study recruited 120 patients who were divided into two groups: group A (guided by health competence) and group $B$ (guided by routine nursing). Whether these 120 patients were randomized or not and the method of randomized grouping were not specified. It is not clear if the study has been approved by the Ethics Committee because the approval information was not provided throughout the study. In the statistical analysis section, the authors did not introduce the basic information of statistical methods and statistical software in detail.
Additionally, since this study was conducted on a small sample, how many patients in both groups had IPH-rated events? If IPH-rated events are infrequent, it may be appropriate to provide a specific number of cases who had IPHrated events. In the results section discussing IPH-related events, the authors mentioned that the internal relationship analysis of the abnormal indicators reveals that hypothermia affects microcirculation metabolism and impairs body immunity. However, we fail to find these data on microcirculation metabolism and body immunity in the results section.

\section{AUTHORS' CONTRIBUTIONS}

MW: Data curation, Formal analysis, Writing - original draft. SW: Conceptualization, Data curation. CL: Conceptualization, Writing - review and editing.

\section{REFERENCE}

1. Wang R, Ping FM, Liu YM, He JL, Du SF, Shi PZ, et al. Effect of competence health cultivation on the prevention and control of inadvertent perioperative hypothermia. Rev Assoc Med Bras (1992). 2020;66(7):924-30. https://doi.org/10.1590/1806-9282.66.7.924

\footnotetext{
'Yingshan People' s Hospital, Department of Anesthesiology - Hubei, China.

${ }^{2}$ Jingmen traditional Chinese medicine hospital, Department of Anesthesiology - Hubei, China.

${ }^{3}$ Jingmen Recovery Hospital, Department of Anesthesiology - Hubei, China.

*Corresponding author: shigaowang886@163.com

Conflicts of interest: the authors declare there are no conflicts of interest. Funding: none.

Received on July 29, 2021. Accepted on August 04, 2021.
} 